\title{
Combined reperfusion therapy to treat cryptogenic acute ischemic stroke during the first trimester of pregnancy: case report and literature review
}

This article was published in the following Dove Press journal:

Therapeutics and Clinical Risk Management

\author{
François Zhu' ${ }^{1,2}$ \\ Benjamin Gory 2,3 \\ Gioia Mione' \\ Lisa Humbertjean' \\ Anne-Laure Derelle ${ }^{2}$ \\ Sébastien Richard ${ }^{1,4}$ \\ 'Department of Neurology, Stroke \\ Unit, University Hospital of Nancy, \\ Nancy Cedex, France; ${ }^{2}$ Department \\ of Diagnostic and Therapeutic \\ Neuroradiology, University Hospital \\ of Nancy, Nancy Cedex, France; \\ ${ }^{3}$ INSERM UI 254, IADI F-54000, \\ Nancy, France; ${ }^{4}$ Centre d'Investigation \\ Clinique Plurithématique Pierre \\ Drouin, CIC-P I 433 INSERM UI I I6, \\ University Hospital of Nancy, \\ Vandoeuvre-lès-Nancy, France
}

Correspondence: François Zhu 29 avenue du Maréchal de Lattre de Tassigny, 54000 Nancy, France

Tel +33383852256

Fax +3338385 II 59

Email f.zhu@chru-nancy.fr

\begin{abstract}
Cerebral infarction due to acute embolism in the large artery during pregnancy is a rare but severe condition threatening both the mother's and child's life. Physicians lack diagnostic and therapeutic guidance to manage this particular situation due to the paucity of published cases. Furthermore, the pathogeny is poorly known, rendering preventive strategies difficult. We describe the case of a young woman presenting cryptogenic acute cerebral infarction during the first trimester of pregnancy who was successfully treated with combined reperfusion therapy. We reviewed the literature to collect data about pathogeny and management. A 28-year-old pregnant woman was diagnosed with acute cerebral infarction due to left middle cerebral artery occlusion at 9 weeks of gestation. Endovascular thrombectomy combined with intravenous thrombolysis allowed cerebral reperfusion leading to a decrease in the National Institute of Health Stroke Score from 13 to 1 at 24 hours. Comprehensive etiological investigation was negative. Anticoagulation therapy with low-molecular-weight heparin was administered as preventive treatment during the pregnancy and postpartum. Neither the mother nor the child experienced any complications: the baby was born by normal vaginal delivery and the outcome was good at 1 year. We identified 21 other cases of patients treated with reperfusion therapies, four of which consisted of endovascular thrombectomy, and only one a combined strategy. Pregnant women with acute cerebral infarction due to arterial occlusion can benefit from combined reperfusion therapy. More cases should be collected to assess treatment in these patients, to understand pathogeny, and propose the best preventive strategy.
\end{abstract}

Keywords: pregnancy, acute cerebral infarction, stroke, rt-PA, thrombolysis, endovascular thrombectomy

Stroke during pregnancy affects 30 women per 100,000 representing a 3-fold higher risk than in young adults overall. Cerebrovascular events in pregnancy are usually related to eclampsia and cerebral venous thrombosis. ${ }^{1,2}$ Cerebral infarction due to acute embolism in a large intracranial artery is rare and has hardly ever been described. Consequently, physicians lack information to diagnose and treat this severe lifethreatening condition for both mother and child. There is a high risk of obstetric and pediatric complications associated with reperfusion therapies. Furthermore, the pathogeny of ischemic stroke during pregnancy remains poorly understood, rendering it difficult to recommend preventive strategies. We report the case of a patient who presented severe cryptogenic cerebral infarction due to occlusion of the left middle cerebral artery (MCA) and who was successfully treated with recombinant tissue plasminogen therapy (rt-PA) and endovascular thrombectomy. Based on our experience 
and a literature review, we describe diagnostic methods, the use of reperfusion therapies, etiological investigations and preventive strategies in this particular context.

\section{Case report}

The patient provided written informed consent for the case details to be published. All data have been anonymized.

\section{History}

A 28-year-old woman with a medical history of miscarriage was admitted in the Verdun Hospital (Lorraine, France) during the first trimester of pregnancy (9 weeks of gestation) because of right motor and sensitive deficit with dysarthria experienced on waking.

\section{Examination}

Neurological examination was performed by the stroke unit of the University Hospital of Nancy 180 minutes later through telemedicine. The patient was found to have a National Institute of Health Stroke Score (NIHSS) of 13; her blood pressure was 93/60 $\mathrm{kPa}$ and heart rate $76 \mathrm{bpm}$. A computed tomography (CT) scan of the brain showed early signs of acute cerebral infarction in the left MCA territory (Figure 1A and B). The Alberta Stroke Program Early CT Score (ASPECTS) was 8. Proximal thromboembolic occlusion of the left MCA was confirmed by cerebral CT angiography (Figure 1C).

\section{Operation}

The patient subsequently underwent intravenous thrombolysis with $70 \mathrm{mg}(0.9 \mathrm{mg} / \mathrm{kg})$ of rt-PA before being transferred to the stroke unit. On arrival (5.5 hours after reporting initial signs) the NIHSS was unchanged. We decided to perform an endovascular thrombectomy.

Conventional angiography was performed on a biplane image-guided system (Innova system GE) with a low dose protocol $\left(686 \mathrm{cGy} / \mathrm{cm}^{2}\right)$ after protecting the patient's abdomen by a lead apron. A Neuron Max 088 catheter (Penumbra Inc., Alameda, CA, USA) was placed in the left internal carotid artery via the femoral artery. Angiography showed persistent occlusion of the proximal segment (M1) of the left MCA (Figure 2). The thrombus was penetrated by a microcatheter RapidTransit ${ }^{\circledR}$ (Codman, Johnson \& Johnson, New Brunswick, NJ, USA) and a microguide wire Terumo 16. A Trevo ProVue (Stryker Neurovascular, Fremont, CA, USA) retriever stent was then delivered through the microcatheter and deployed inside the thrombus for 3 minutes. A Sofia $^{\circledR}$ 6F (MicroVention, Aliso Viejo, CA, USA) aspiration catheter was advanced to the level of the occlusion. Continuous aspiration was performed for 30 seconds using a Penumbra aspiration pump and the system was then slowly removed under aspiration. A 6-mm red clot was taken out from between the stent retriever and the aspiration catheter (Figure 3). Angiography showed successful recanalization of more than half of the left MCA territory (Thrombolysis in Cerebral Infarction [TICI] scale grade IIb). A slight spasm was observed on the proximal segment (M1) (Figure 4). The NIHSS score immediately decreased to 3 and then to 1 by the 24-hour assessment with limited infarction of the corpus striatum on the control CT scan (Figure 5).

\section{Pathological findings}

Comprehensive etiological investigations did not reveal any cause of the cerebral infarction. It was thus classified
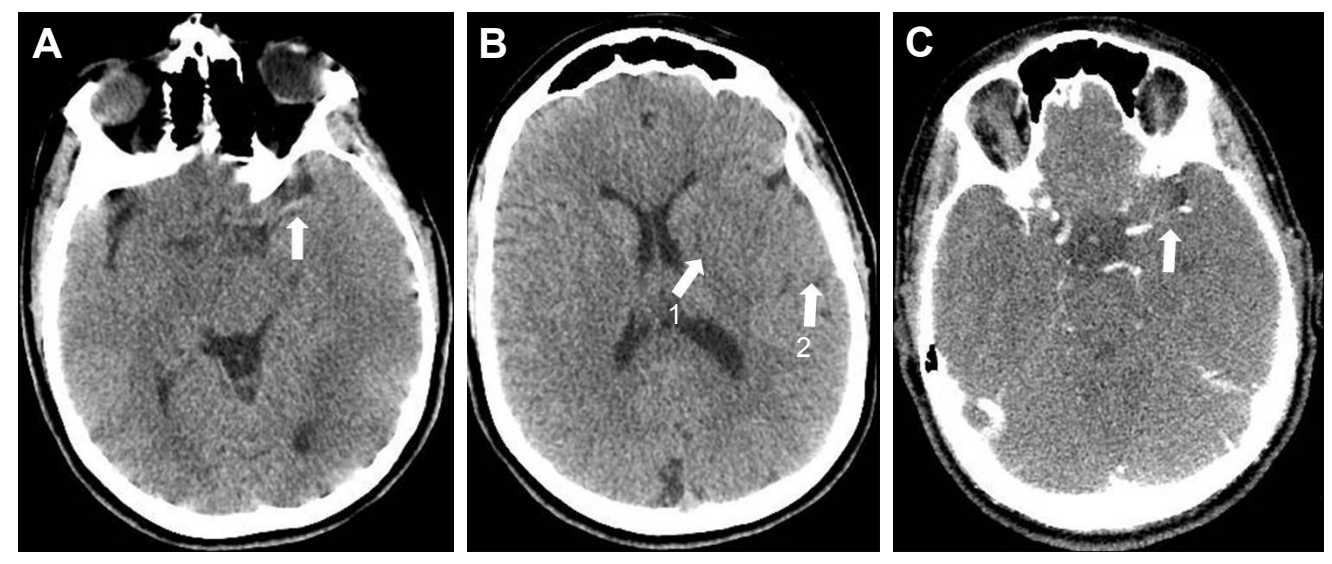

Figure I Cerebral CT scan showing spontaneous hyperdensity in the left middle cerebral artery proximal segment MI (A), lentiform nucleus obscuration (B, arrow I), loss of gray-white matter discrimination (B, arrow 2), and proximal occlusion of the left middle cerebral artery on CT angiography (C).

Notes: (A) Cerebral CT scan showing spontaneous hyperdensity in the left middle cerebral artery proximal segment MI (white arrow). (B) Proximal occlusion of the left middle cerebral artery on CT angiography (white arrow).

Abbreviation: CT, computed tomography. 

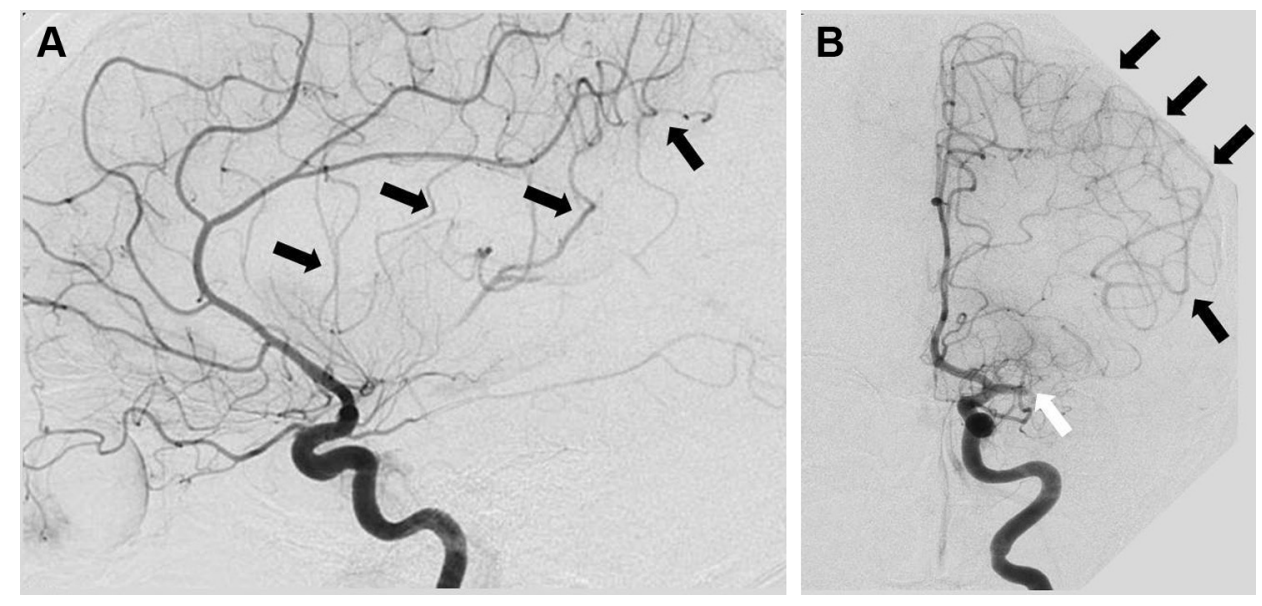

Figure 2 Initial left internal carotid angiogram (lateral [A], and anteroposterior [B] views) showing proximal occlusion of the left middle cerebral artery (white arrow), lack of perfusion in the respective territory, and collateral flow from the left anterior cerebral artery through leptomeningeal anastomoses (black arrows).

as cryptogenic. A detailed medical interview revealed no history of cardiovascular disease (personal or familial), drug consumption, or recent headache. Cervical and transcranial Doppler ultrasonography were normal. Cervical arteries were also examined during conventional angiography with no sign of dissection. Electrocardiogram and 72-hour cardiac monitoring did not detect arrhythmia and blood pressure levels were normal. Transoesophageal echocardiography failed to find any thrombus or patent foramen ovale. Blood tests did not reveal any prothrombotic anomalies: the hemoglobin level was $12.6 \mathrm{~g} / \mathrm{dL}$, the platelet count was $312 \mathrm{G} / \mathrm{L}$, C-reactive protein level was $<5 \mathrm{mg} / \mathrm{L}$, the low-density lipoprotein-cholesterol level was $1.89 \mathrm{mmol} / \mathrm{L}$, the preprandial blood glucose level was $4.83 \mathrm{mmol} / \mathrm{L}$, and the homocysteine level was normal at $12.6 \mu \mathrm{mol} / \mathrm{L}$. All the coagulation tests including prothrombin time, activated partial thromboplastin time, lupus anticoagulant and anticardiolipin antibody were normal. Anticoagulation factor activities were normal for protein $\mathrm{S}$, protein $\mathrm{C}$ and antithrombin (respectively at 78,

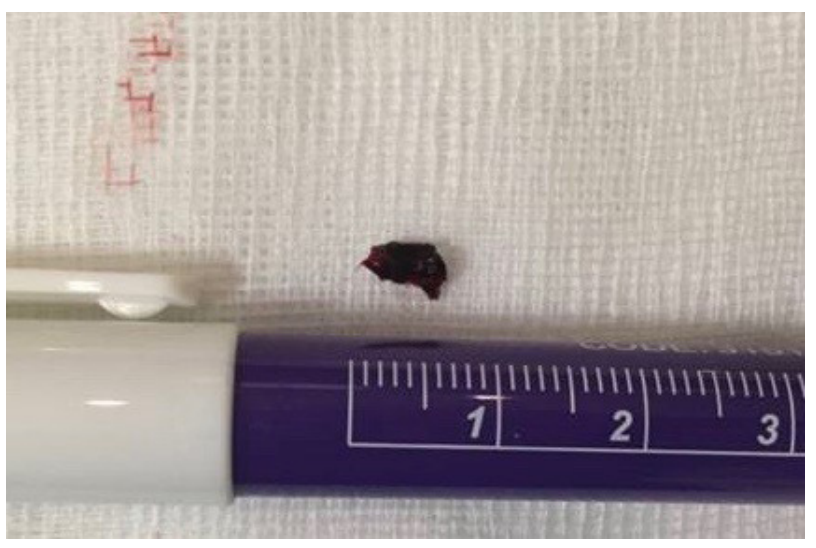

Figure 3 The $6 \mathrm{~mm}$ red clot removed from left middle cerebral artery following endovascular thrombectomy.
99, and 91\%). No activated protein $\mathrm{C}$ resistance or mutation of the prothrombin gene were found.

\section{Postoperative course}

Obstetric examination and fetal ultrasound showed that neither the mother nor the fetus had experienced any complications. Anticoagulation therapy was initiated as a preventive strategy with low-molecular-weight heparin (tinzaparin 175 anti-Xa IU/kg/day) and administered up to 35 weeks of gestation, when a daily dose of enoxaparin (4,000 anti-Xa IU) was started. The patient gave birth at a gestational age of 39 weeks and 2 days to a healthy infant of $3.8 \mathrm{~kg}$ by normal vaginal delivery with no complications. The Apgar score at 5 minutes was 7/10 and the umbilical cord arterial blood $\mathrm{pH}$ was 7.35. Enoxaparin was discontinued after postpartum and replaced by a daily dose of $160 \mathrm{mg}$ of aspirin. Both the mother and child had a normal medical examination at 1 year.

\section{Discussion}

We describe the diagnosis and management of an acute cryptogenic cerebral infarction due to left MCA occlusion in a woman during the first trimester of pregnancy. The patient was successfully treated by combined reperfusion therapy.

The first issue that arises for a woman with stroke symptoms during the first trimester of pregnancy is the use of diagnostic cerebral imaging. We used a cerebral CT scan with iodine injection for intracranial angiography. According to the French public expert authority in nuclear and radiological risks (IRSN), the uterus is exposed to less than $0.1 \mathrm{mGy}$ during this examination, which is under the teratogenic dose range. ${ }^{3,4}$ However, most centers in France 

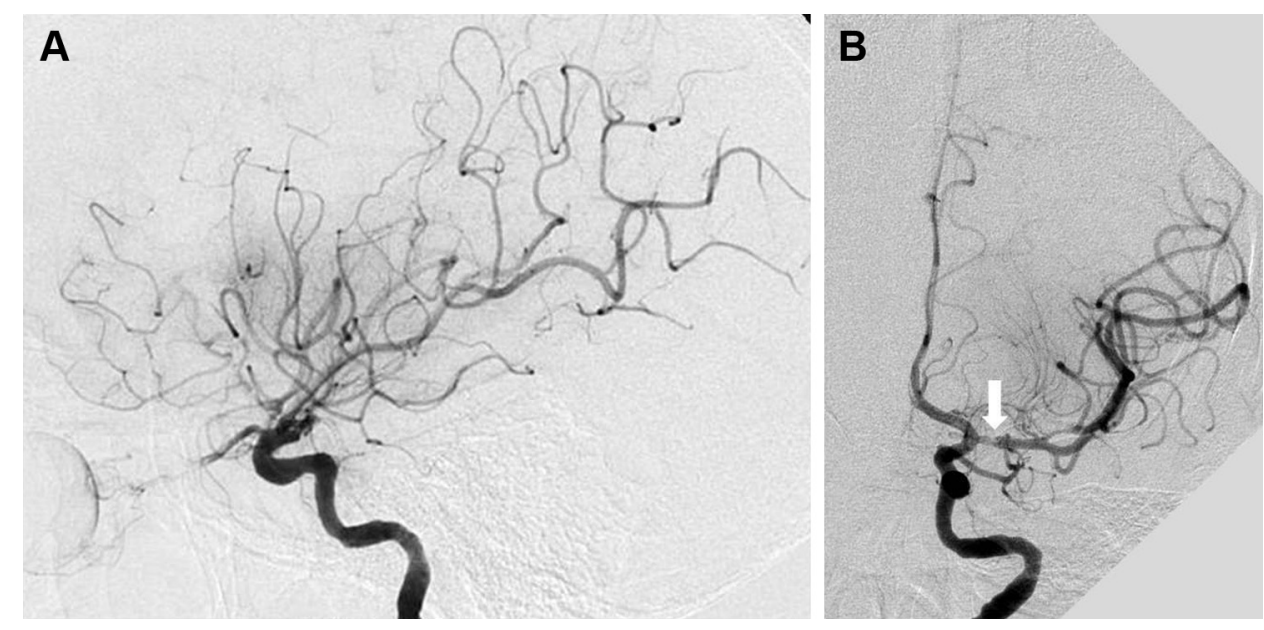

Figure 4 Left internal carotid artery angiogram (lateral [A], and anteroposterior [B] views) showing recanalization (Thrombolysis in Cerebral Infarction [TICI] scale grade Ilb), and slight vasospasm (white arrow) of the left middle cerebral artery following mechanical thrombectomy.

prefer cerebral MRI to diagnose acute stroke and determine reperfusion therapies. This would have allowed a better estimation of the time of the stroke onset. ${ }^{5}$ Ray et al compared the outcome in 1,737 women who underwent MRI during the first trimester of pregnancy with an unexposed cohort of more of 1,400,000 patients. They found no significant increased risk of fetal or early infant death, congenital anomalies, neoplasms, vision or hearing loss in the children by the age of 4 years. ${ }^{6}$ MRI has also been shown to be safe in women in the second and third trimesters of pregnancy. ${ }^{7}$ Nevertheless, gadolinium injection should be avoided throughout pregnancy because of a significant risk of perinatal mortality and a broad set of rheumatological and infiltrative skin diseases related to nephrogenic systemic fibrosis. ${ }^{6}$ The intracranial arteries can be explored through three-dimensional time-offlight sequence and cerebral perfusion through arterial spin labeling sequence without contrast agent injection. Unlike a cerebral CT-scan, MRI does not require additional irradiation or injections of iodine inherent to conventional angiography for endovascular thrombectomy. A low dose protocol with tight collimation on angiogram should be used allowing a $50 \%$ reduction in irradiation compared to usual procedures and provides satisfactory, through reduced image resolution. ${ }^{8}$ The infant should be examined for neonatal hypothyroidism at 3 days due to the iodine injection even if this would appear to be a rare complication. ${ }^{9}$

Physicians are reluctant to use rt-PA to treat acute cerebral infarction in pregnant women due to the risk of obstetric hemorrhage and the unknown effect on fetal development, especially during the first trimester. However, literature argues in favor of rt-PA safety during pregnancy. Data from animal models suggest that rt-PA does not pass the placental barrier due to its large molecular size, and no teratogenic effects have been observed..$^{10}$ Gartman reviewed 231 cases of thrombolytic agent use in patients to treat systemic arterial or venous thrombosis during pregnancy and found
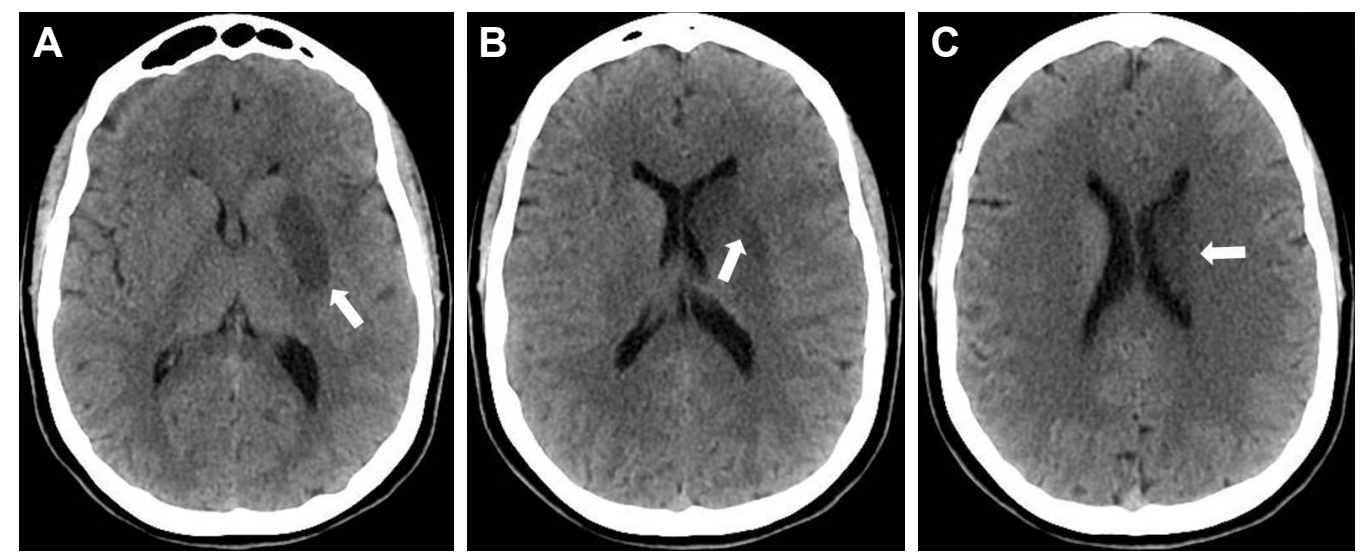

Figure 5 Control cerebral CT-scan performed 24 hours after reperfusion therapies showing a left lenticulostriate (A, B) and caudate (C) infarct (white arrows). Abbreviation: CT, computed tomography. 
no significant increased risk of complication compared to a non-pregnant population. ${ }^{11}$ Tversky et al and Reining-Festa et al identified 17 cases of patients with acute cerebral infarction during pregnancy treated with rt-PA (intravenous or intraarterial) in their systematic literature review. ${ }^{12,13} \mathrm{We}$ found another case where rt-PA was used for combined reperfusion therapy. ${ }^{14}$ In all, two medical terminations of pregnancy have been reported, including one due to a lethal chromosomal anomaly. ${ }^{15}$ One patient presented an intrauterine hematoma, which was successfully drained. ${ }^{15}$ Three patients experienced cerebral hemorrhagic complications after rt-PA: two presented asymptomatic deep hematoma, ${ }^{16,17}$ and another died but in the context of arterial dissection related to angioplasty. ${ }^{15}$ Finally, international guidelines incite physicians to consider intravenous thrombolysis during pregnancy "when the anticipated benefits of treating moderate to severe stroke outweigh the anticipated increased risks of uterine bleeding." 18

Endovascular thrombectomy now forms an integral part of reperfusion therapies to treat proximal occlusion of cerebral arteries. ${ }^{19}$ Four cases during pregnancy have recently been reported in the literature. Aaron et al described two cases of MCA occlusion treated with endovascular thrombectomy only through aspiration by the Penumbra system. ${ }^{20}$ Bhogal et al reported a case of MCA occlusion treated by stent retriever thrombectomy and a basilar artery occlusion treated by combined reperfusion therapy. ${ }^{14}$ The outcome was good for both the mothers and children in these four cases, emphasizing the benefit of this treatment approach in this specific population. Endovascular thrombectomy alone could be an interesting strategy in acute ischemic stroke management during pregnancy as it prevents the mother and fetus from being exposed to rt-PA and the inherent risks. More broadly, some studies have shown that endovascular therapy provides a higher rate of recanalization, thus improving outcome in young patients and supporting aggressive interventional strategies in this population. ${ }^{21}$ Nevertheless, to date, the effectiveness of endovascular thrombectomy during the first hours after stroke onset has only been demonstrated in combination with intravenous thrombolysis. ${ }^{22}$ In our case we were able to initiate intravenous thrombolysis early in a local hospital through telestroke as the patient was approximately 2 hours from an endovascular thrombectomy capable center.

After managing the acute phase, it is crucial to undertake etiological investigations to understand the stroke mechanism so as to initiate a prevention strategy. Most etiologies described in literature pertain to a young population with stroke: cardiac valve diseases (especially rheumatologic valve disease), coagulopathy, and patent foramen ovale. . $13,23,24^{2}$ The most frequent etiology of cerebral infarction in this population is cervical artery dissection. The risk of cervical artery dissection is accrued during pregnancy and postpartum by modifications of media due to hormonal changes. ${ }^{23,25}$ Pregnant women presenting with stroke should thus undergo the same etiological investigation as the general young population with stroke, paying particular attention to exploration of cervical and intracranial arteries, transoesophageal echocardiography, and detection of biological prothrombotic agents (platelet count, lupus anticoagulant and anticardiolipin antibody). Most cerebrovascular events occurring during pregnancy are related to eclampsia and cerebral venous thrombosis, both of which cause cerebral infarction and hematomas. ${ }^{2}$ Several other etiologies have been reported such as amniotic fluid embolism, postpartum angiopathy and ovarian stimulation. ${ }^{18,26}$ Comprehensive etiological investigation failed to find any of these causes in our patient (including absence of patent foramen ovale), which led us to classify this cerebral infarction as cryptogenic.

Although cryptogenic strokes can occur in the general young population, our case raises the question of the role of pregnancy in the pathogeny of cerebral infarction. Besides the hemodynamic changes brought about by elevated blood volume and cardiac flow during pregnancy, ${ }^{27}$ some reports seem to incriminate a prothrombotic state but without convincing evidence. Partly because of hormonal modifications, ${ }^{28}$ pregnancy is associated with major changes in hemostasis due to an increase in most thrombotic agents (factors VIII, X, XII, von Willebrand, and ristocetin cofactors) combined with a decrease in the quality of natural anticoagulants such as protein S. Fibrinolytic activity is decreased due to the production of plasminogen activator inhibitor by the placenta. ${ }^{29}$ However, these abnormalities are more likely to cause venous thromboembolism, and have been described during advanced stages of pregnancy or during the postpartum..$^{23,29}$

In view of the unknown origin of the stroke in our patient and the possible implication of a prothrombotic state as suggested by literature, we decided to administer anticoagulation therapy to prevent recurrence during pregnancy and postpartum even though this approach remains debatable. ${ }^{30}$ Several studies are currently underway to determine whether anticoagulation or antiplatelet therapy is the best preventive strategy in cases such as ours. ${ }^{31}$ While use of heparin and low-dose aspirin use is allowed during pregnancy, oral anticoagulants are not recommended during the first and third trimesters due to a risk of malformation. ${ }^{32}$ Currently, there are no data reporting the use of direct oral anticoagulants and clopidogrel. A cesarean section is preferable in patients with obstetric complication or recent cervical artery dissection only, to prevent worsening during labor from pushing. ${ }^{33}$ 
Finally, a French multicenter study has shown that stroke recurrence during subsequent pregnancies is rare. ${ }^{34}$

\section{Conclusion}

Management of acute cerebral infarction in pregnant women should not differ from that in the general population. Adjustments are possible to minimize irradiation during the diagnostic phase. Combined reperfusion therapy remains the best strategy to restore intracranial reperfusion, without compromising safety, and consequently to ensure good outcome for both mother and child. Nevertheless, it is necessary to collect more cases to assess these practices and especially to understand the pathogeny of stroke during pregnancy.

\section{Disclosure}

The authors report no conflict of interest concerning the materials or methods used in this study or the findings specified in this paper.

\section{References}

1. James AH, Bushnell CD, Jamison MG, Myers ER. Incidence and risk factors for stroke in pregnancy and the puerperium. Obstet Gynecol. 2005;106(3):509-516.

2. Swartz RH, Cayley ML, Foley N, et al. The incidence of pregnancyrelated stroke: A systematic review and meta-analysis. Int J Stroke. 2017;12(7):687-697.

3. French public expert authority in nuclear and radiological risks (IRSN). [Delivered doses to patients in Computed Tomography and conventional radiology] Doses délivrées aux patients en scanographie et en radiologie conventionnelle Rapport DRPH/SER n²010-12. Available from: http:// www.irsn.fr/FR/expertise/rapports_expertise/Documents/radioprotection/IRSN-Rapport-dosimetrie-patient-2010-12.pdf. Accessed August 23, 2018. French.

4. de Santis M, di Gianantonio E, Straface G, et al. Ionizing radiations in pregnancy and teratogenesis: a review of literature. Reprod Toxicol. 2005;20(3):323-329.

5. Thomalla G, Cheng B, Ebinger M, et al. DWI-FLAIR mismatch for the identification of patients with acute ischaemic stroke within 4:5 h of symptom onset (PRE-FLAIR): a multicentre observational study. Lancet Neurol. 2011;10(11):978-986.

6. Ray JG, Vermeulen MJ, Bharatha A, Montanera WJ, Park AL. Association between MRI exposure during pregnancy and fetal and childhood outcomes. JAMA. 2016;316(9):952-961.

7. Strizek B, Jani JC, Mucyo E, et al. Safety of MR imaging at $1.5 \mathrm{~T}$ in fetuses: a retrospective case-control study of birth weights and the effects of acoustic noise. Radiology. 2015;275(2):530-537.

8. Yi HJ, Sung JH, Lee DH, Kim SW, Lee SW. Analysis of radiation doses and dose reduction strategies during cerebral digital subtraction angiography. World Neurosurg. 2017;100:216-223.

9. Chauvet $\mathrm{P}$, Terral D, Colombier M, et al. [Iodinated contrast in pregnant women and neonatal thyroid function] Injection de produit de contraste iodé en cours de grossesse et fonction thyroïdienne néonatale. Gynecol Obstet Fertil. 2016;44(12):685-689. French.

10. Tanaka M. Study in rats treated intravenously with GMK-527 (II) teratogenicity study in rats with GMK-527. Yakuri Chiryo. 1988;16:93-106.

11. Gartman EJ. The use of thrombolytic therapy in pregnancy. Obstet Med. 2013;6(3):105-111.

12. Tversky S, Libman RB, Reppucci ML, Tufano AM, Katz JM. Thrombolysis for ischemic stroke during pregnancy: a case report and review of the literature. J Stroke Cerebrovasc Dis. 2016;25(10):e167-e170.
13. Reining-Festa A, Földy D, Coulibaly-Wimmer M, Eischer L, Heger M, Fertl E. Intravenous thrombolysis of stroke in early pregnancy: a case report and review of the literature. J Neurol. 2017;264(2): 397-400.

14. Bhogal P, Aguilar M, Almatter M, Karck U, Bäzner H, Henkes H. Mechanical thrombectomy in pregnancy: report of 2 cases and review of the literature. Interv Neurol. 2017;6(1-2):49-56.

15. Murugappan A, Coplin WM, Al-Sadat AN, et al. Thrombolytic therapy of acute ischemic stroke during pregnancy. Neurology. 2006;66(5): 768-770.

16. Dapprich M, Boessenecker W. Fibrinolysis with alteplase in a pregnant woman with stroke. Cerebrovasc Dis. 2002;13(4):290.

17. Elford K, Leader A, Wee R, Stys PK. Stroke in ovarian hyperstimulation syndrome in early pregnancy treated with intra-arterial rt-PA. Neurology. 2002;59(8):1270-1272.

18. Demaerschalk BM, Kleindorfer DO, Adeoye OM, et al. Scientific rationale for the inclusion and exclusion criteria for intravenous alteplase in acute ischemic stroke: a statement for healthcare professionals from the American Heart Association/American Stroke Association. Stroke. 2016;47(2):581-641.

19. Nogueira RG, Jadhav AP, Haussen DC, et al. Thrombectomy 6 to 24 hours after stroke with a mismatch between deficit and infarct. N Engl J Med. 2018;378(1):11-21.

20. Aaron S, Shyamkumar NK, Alexander S, et al. Mechanical thrombectomy for acute ischemic stroke in pregnancy using the penumbra system. Ann Indian Acad Neurol. 2016;19(2):261-263.

21. Chalouhi N, Tjoumakaris S, Starke RM, et al. Endovascular stroke intervention in young patients with large vessel occlusions. Neurosurg Focus. 2014;36(1):E6.

22. Bracard S, Ducrocq X, Mas JL, et al. Mechanical thrombectomy after intravenous alteplase versus alteplase alone after stroke (THRACE): a randomised controlled trial. Lancet Neurol. 2016;15(11): 1138-1147.

23. Ulrich N, Johnson A, Jodry D, Dola C, Martin-Schild S, El Khoury R. Resolution of internal carotid dissection with middle cerebral artery occlusion in pregnancy. Case Rep Neurol Med. 2015;2015:1-5.

24. Skidmore FM, Williams LS, Fradkin KD, Alonso RJ, Biller J. Presentation, etiology, and outcome of stroke in pregnancy and puerperium. J Stroke Cerebrovasc Dis. 2001;10(1):1-10.

25. Barrett JM, van Hooydonk JE, Boehm FH. Pregnancy-related rupture of arterial aneurysms. Obstet Gynecol Surv. 1982;37(9):557-566.

26. Frontera JA, Ahmed W. Neurocritical care complications of pregnancy and puerperum. J Crit Care. 2014;29(6):1069-1081.

27. Lund CJ, Donovan JC. Blood volume during pregnancy. Significance of plasma and red cell volumes. Am J Obstet Gynecol. 1967;98(3): 394-403.

28. Sattar N, Greer IA, Rumley A, et al. A longitudinal study of the relationships between haemostatic, lipid, and oestradiol changes during normal human pregnancy. Thromb Haemost. 1999;81(1):71-75.

29. Thornton P, Douglas J. Coagulation in pregnancy. Best Pract Res Clin Obstet Gynaecol. 2010;24(3):339-352.

30. Helms AK, Drogan O, Kittner SJ. First trimester stroke prophylaxis in pregnant women with a history of stroke. Stroke. 2009;40(4): 1158-1161.

31. Hart RG, Catanese L, Perera KS, Ntaios G, Connolly SJ. Embolic stroke of undetermined source: a systematic review and clinical update. Stroke. 2017;48(4):867-872.

32. Bates SM, Greer IA, Middeldorp S, Veenstra DL, Prabulos A-M, Vandvik PO. VTE, thrombophilia, antithrombotic therapy, and pregnancy. Chest. 2012;141(2):e691S-e736S.

33. Reinhard M, Munz M, von Kannen AL, Griesser-Leute HJ, Dittrich R, Engelter ST. Risk of recurrent cervical artery dissection during pregnancy, childbirth and puerperium. Eur J Neurol. 2015;22(4): 736-739.

34. Lamy C, Hamon JB, Coste J, Mas JL. Ischemic stroke in young women: risk of recurrence during subsequent pregnancies. French Study Group on Stroke in Pregnancy. Neurology. 2000;55(2):269-274. 
Therapeutics and Clinical Risk Management

Dovepress

\section{Publish your work in this journal}

Therapeutics and Clinical Risk Management is an international, peerreviewed journal of clinical therapeutics and risk management, focusing on concise rapid reporting of clinical studies in all therapeutic areas outcomes, safety, and programs for the effective, safe, and sustained use of medicines. This journal is indexed on PubMed Central, CAS,
EMBase, Scopus and the Elsevier Bibliographic databases. The manuscript management system is completely online and includes a very quick and fair peer-review system, which is all easy to use. Visit http://www.dovepress.com/testimonials.php to read real quotes from published authors.

Submit your manuscript here: http://www.dovepress.com/therapeutics-and-clinical-risk-management-journal 